\title{
ON QUANTUM MECHANICS OF MANY-BODY SYSTEMS WITH DILATION-ANALYTIC POTENTIALS
}

\author{
BY I. M. SIGAL
}

Communicated by R. G. Douglas, May 4, 1977

1. In the paper [2] we have proved the asymptotic completeness of many particle nonrelativistic quantum systems with potentials, $V_{\alpha}$, satisfying the direct restriction

$$
\left|v_{\alpha}(k)\right|+|h|^{-\nu}\left|v_{\alpha}(k+h)-v_{\alpha}(k)\right| \leqslant C(1+|k|)^{-\eta}, \nu>\frac{1}{2}, \eta>\frac{3}{2},
$$

where $v_{\alpha}(k)=\int V_{\alpha}(x) e^{-i k x} d x$, and the two indirect restrictions:

(i) No compound system has eigenvalues embedded in its continuous spectrum;

(ii) No compound system has quasibound states (at its thresholds). The definitions of a compound system and a quasibound state will be given later. The indirect restrictions are discussed in [2] and it has been stated there without proof that they are satisfied for "almost all" dilation analytic short range (analytic short range potentials) potentials. The aim of this note is to present the precise statements of the results mentioned above and some ideas of their proofs. We include also some other related results. Complete proofs will be published elsewhere.

2. Henceforth $H$ is the Hamiltonian of an $n$-body system in its center-ofmass frame.

THEOREM 1. Let $V_{i j}$ satisfy the Combes conditions and besides let $V_{i j}(\theta)$ $\forall \theta \in 0$ obey

$$
\int\left|v_{\alpha}(k ; \theta)\right|^{m}(1+|k|)^{\eta m} d k<C, m>3, \eta>\frac{3}{2}\left(1-\frac{2}{m}\right)
$$

Then the set of all $g \in \mathbf{R}^{n(n-1) / 2}$ such that $H(g)=H_{0}+\Sigma_{i<j} g_{i j} V_{i j}$ has no quasibound states is nondense in $\mathrm{R}^{n(n-1) / 2}$.

Theorem 2. Let $V_{i j}$ satisfy the assumptions of Theorem 1 and let no compound system have quasibound states (at its thresholds). Then the number of bound states of $H$ is finite and the resonances of $(H, D(O))^{1}$ have no accumulation points on the real axis.

AMS (MOS) subject classifications (1970). Primary 47A40, 81A48.

${ }^{1}$ For the definition of the set $D(0)$ see [1]. The resonances of $(H, D(0))$ are nonreal eigenvalues of the Combes-Balslev family $H(\theta)([1])$.

Copyright $\odot$ 1978, American Mathematical Society 
THEOREM 3. Let the assumptions of Theorem 2 be satisfied. There are a potential $W=1 / 2 \Sigma W_{i j}$, where $W_{i j}$ are delation analytic, smooth and fast decreasing in $\mathbf{R}^{3}$, and a number $\epsilon_{0}>0$, such that $H+\epsilon W, 0<|\epsilon| \leqslant \epsilon_{0}$, have no eigenvalues embedded into their continuous spectra.

THEOREM 4. Under the assumptions of Theorem 2 and the additional restrictions

$$
\begin{aligned}
\int\left|\Delta(h) D^{[\nu]} v_{\alpha}(k ; \theta)\right|^{m}(1 & +|k|)^{\eta m}|k|^{m-1} d k \\
& \leqslant C|h|^{\nu-[\nu]}, 2 \leqslant m<\infty, \nu>\frac{2}{m}, \eta>\frac{1}{2}-\frac{2}{m},
\end{aligned}
$$

and no compound system has eigenvalues embedded into its continuous spectrum, the system $\left\{\Omega_{a, m}^{ \pm}\right\}$of channel wave operators for $H$ is complete.

3. Now we give some definitions needed later. By lower case roman letters $\underline{a}, \underline{b}, \ldots$, we denote different decompositions of the set $\{1, \ldots, n\}$ into disjoint, nonempty subsets, $C_{i}$, such that $U C_{i}=\{1, \ldots, n\}$. The number of subsets in a partition $\underline{a}$ is denoted by $k(a)$. We assign to every decomposition $\underline{a}$ the operator $H^{a}=\bigoplus_{C_{i} \in a} H^{C}$, where $H^{C}$ is the Hamiltonian for a cluster $C$ in its center-ofmass frame, $H=H^{a}$ for $a=(1, \ldots, n)$. The systems described by the Hamiltonians $H^{a}, k(a)>1$, will be called compound systems. We put $\sigma_{p}\left(H^{a}\right)=\{0\}$ for $a=\{(1), \ldots,(n)\} . \tau(H)=\bigcup_{k(a)>1} \sigma_{p}\left(H^{a}\right)$ is the threshold set for $H$. All notions defined above for $H$ can be redefined for the Balslev-Combes family $H(\theta)$. Set $G_{\theta}=\mathbf{C l}\left(\tau(H(\theta))+\overline{\mathbf{R}}^{+} e^{-2 \theta}\right)$.

4. The proofs of the theorems are based on the study of the family $H(\theta)$ for given $H$. To this end we use an approach based on a regularization of $H(\theta)$ $-z$. Central to this approach is the following statement which expresses properties of $H(\theta)$ in terms of a regularizer for $H(\theta)-z$ :

Proposition 3. Let there exist scales Banach spaces $B_{s}$ and $\hat{B}_{s}$, a linear map $\pi(z, \theta)$ of $\hat{B}_{s}$ and an operator-valued function $F(z, \theta), z \in G_{\theta}$, defined on $B_{s}$ such that

(a) $B_{s} \subset H, \pi(z, \theta) \hat{B}_{s} \subset D(H), \exists s_{0}: \pi(z, \theta) \hat{B}_{s_{0}}=D(H)$ for $z \in G_{0}$

(b) $F(z, \theta)$ can be represented in the form

$$
F(z, \theta)=\pi(z, \theta) \hat{F}(z, \theta),
$$

where $\hat{F}(z, \theta), z \in G_{\theta}$, is a bounded operator from $B_{s}$ into $\hat{B}_{s}$;

(c) $\forall z \in G_{\theta}, F(z, \theta)$ has an inverse such that $F(z, \theta)^{-1} \pi(z, \theta)$ is bounded from $\hat{B}_{s}$ into $B_{s}$;

(d) The operator $A(z, \theta) \equiv(H(\theta)-z) F(z, \theta)-1$ has a compact power in $\boldsymbol{B}_{\boldsymbol{s}}$.

Then $\sigma_{e s}(H(\theta)) \subset \subset \backslash G_{\theta}$. Hence eigenvalues of $H(\theta)$ can accumulate only to points of $\left(\bigcap_{\varphi<\varphi^{\prime}<\varphi+\epsilon} \partial G_{\theta^{\prime}}\right) \psi\left(\bigcap_{\varphi-\epsilon<\varphi^{\prime}<\varphi} \partial G_{\theta^{\prime}}\right)$ ( $\epsilon$ is sufficiently small). Note that $\tau(H(\theta))=\bigcap_{\left|\varphi^{\prime}-\varphi\right|<\epsilon} \partial G_{\theta^{\prime}}$. Here $\varphi=\operatorname{Im} \theta, \varphi^{\prime}=\operatorname{Im} \theta^{\prime}$. 
If in addition to (a)-(d) the following condition is satisfied for every $z_{0} \in$ $\left(\bigcap_{\varphi \leqslant \varphi^{\prime} \leqslant \varphi+\epsilon} \partial G_{\theta^{\prime}}\right) \cup\left(\bigcap_{\varphi-\epsilon \leqslant \varphi^{\prime} \leqslant \varphi^{\prime}} \partial G_{\theta^{\prime}}\right):$

(e) $\exists s_{1}: \pi\left(z_{0}, \theta\right) \hat{B}_{s_{1}}$ the space dual to $B_{s_{1}}$;

(e) $\hat{F}(z, \theta)$ and $F(z, \theta)^{-1} \pi(z, \theta)$ are weakly continuous and $A(z, \theta)$ is continuous in the operator topology as $z$ approaches $z_{0}$, then eigenvalues of $H(\theta)$ have no finite accumulation points.

If in addition to (a)-(e) the following conditions are satisfied:

(f) As $z$ approaches $\partial G_{\theta}, \hat{F}(z, \theta)$ and $F(z, \theta)^{-1} \pi(z, \theta)$ have strong limits and $A(z, \theta)$ raised to some power has a limit in the operator norm,

(g) $\forall z \in \mathbf{C}, A(z, \theta)$ is analytic in $\theta \in \Omega_{z} \equiv\left\{\theta \in O, z \in G_{\theta}\right\}$ and weakly continuous $\bar{\Omega}_{z}$.

then $R(z, \theta)=(H(\theta)-z)^{-1}$ can be represented in the form

$$
R(z, \theta)=P(z, \theta)+\pi(z, \theta) \hat{R}(z, \theta),
$$

where $P(z, \theta)$ is the discrete part of $R(z, \theta)$ and $\hat{R}(z, \theta)$ is a bounded operator from $B_{s}$ into $\hat{B}_{s}$ for all $z \in G_{\theta}$ and has strong limits as $z$ approaches $\partial G_{\theta}$ with the possible exception of neighborhoods of two-cluster thresholds. Here $\theta$ can be any number from $O$ with the possible exception of a finite number of lines parallel to the real axis.

5. Now we can give a definition of quasibound states. We say that a system described by $H$ has a quasibound state at a two-cluster threshold $z_{0}$, only if the equation

$$
f+A\left(z_{0}, \theta\right) f=0,
$$

where $A(z, \theta)$ satisfies (a)-(f) of Proposition 1, has a nontrivial solution $f_{0}$ in $B_{s}$ such that $F\left(z_{0}, \theta\right) f_{0} \notin D(H)$.

In the same way one defines quasibound states for compound systems.

Note that $F\left(z_{0}, \theta\right) f_{0}$ is a solution of the equation $H(\theta) f=z_{0} f$. Using regularizers we can prove that if $H(\theta) f=z f$ has a solution in $\pi\left(z_{0}, \theta\right) \hat{B}_{s}$ for $z \in G_{\theta}$ then this solution belongs to $D(H)$, i.e. it is an eigenvector of $H(\theta)$.

We built the regularizer $F(z, \theta)$ using the potentials and the resolvents of the compound systems. Therefore its study can be conducted by induction.

\section{REFERENCES}

1. E. Balslev and J. M. Combes, Spectral properties of many-body Schrödinger operators with dilatation-analytic interactions, Comm. Math. Phys. 22 (1971), 280-294.

2. I. M. Sigal, Mathematical foundations of quantum scattering theory for multiparticle systems, Mem. Amer. Math. Soc. (in press).

Institut fur Theoretische Physik, ETH, Zurich, Switzerland 